\title{
Construct new type solutions for the fractional Schrödinger equation
}

Yuan Lin ${ }^{1}$ and Weiming Liư ${ }^{1 *}$

\author{
"Correspondence: \\ whu.027@163.com \\ 'School of Mathematics and \\ Statistics, Hubei Normal University, \\ Huangshi, 435002, P.R. China
}

\begin{abstract}
This paper is devoted to studying the following nonlinear fractional problem:

$$
\left\{\begin{array}{l}
(-\Delta)^{s} u+u=K(|x|) u^{p}, \quad u>0, x \in \mathbb{R}^{N}, \\
u(x) \in H^{s}\left(\mathbb{R}^{N}\right),
\end{array}\right.
$$

where $N \geq 3,0<s<1,1<p<\frac{N+2 s}{N-2 s}, K(|x|)$ is a positive radical function. We constructed infinitely many non-radial solutions of the new type which have a more complex concentration structure for (0.1).
\end{abstract}

MSC: 35B09; 35J10;47J30

Keywords: Fractional Schrödinger equation; Lyapunov-Schmidt reduction method; New type solutions

\section{Introduction and main result}

In this paper, we focus on the following fractional problem:

$$
\left\{\begin{array}{l}
(-\Delta)^{s} u+u=K(|x|) u^{p}, \quad u>0, x \in \mathbb{R}^{N}, \\
u(x) \in H^{s}\left(\mathbb{R}^{N}\right),
\end{array}\right.
$$

where $N \geq 3,0<s<1,1<p<\frac{N+2 s}{N-2 s}, K(|x|)$ is a positive radical function. Problem (1.1) arises from the study of time-independent waves $\psi(x, t)=e^{-i E t} u(x)$ of the following nonlinear fractional Schrödinger equation:

$$
i \frac{\partial \psi}{\partial t}=(-\Delta)^{s} \psi+P(x) \psi-|\psi|^{p-1} \psi, \quad x \in \mathbb{R}^{N}, t>0
$$

In (1.1), the fractional Laplacian operator $(-\Delta)^{s}$ is defined as

$$
(-\Delta)^{s} u(x)=C_{N, s} P . V \cdot \int_{\mathbb{R}^{N}} \frac{u(x)-u(y)}{|x-y|^{N+2 s}} d y,
$$

where $C_{N, s}$ is some normalization constant and P.V. stands for the Cauchy principle value. The fractional Schrödinger equation was discovered by Laskin [23, 24] as a result of ex-

(c) The Author(s) 2021. This article is licensed under a Creative Commons Attribution 4.0 International License, which permits use, sharing, adaptation, distribution and reproduction in any medium or format, as long as you give appropriate credit to the original author(s) and the source, provide a link to the Creative Commons licence, and indicate if changes were made. The images or other third party material in this article are included in the article's Creative Commons licence, unless indicated otherwise in a credit line to the material. If material is not included in the article's Creative Commons licence and your intended use is not permitted by statutory regulation or exceeds the permitted use, you will need to obtain permission directly from the copyright holder. To view a copy of this licence, visit http://creativecommons.org/licenses/by/4.0/. 
tending the Feynman path integral from the Brownian-like paths to Lévy-like quantum mechanical paths. This problem has a strong physical background and it has frequently been studied by a lot of researchers.

Problem (1.1) is a typical case of the equation

$$
(-\Delta)^{s} u+V(x) u=f(x, u), \quad u>0, x \in \mathbb{R}^{N} .
$$

For a subcritical power, under suitable conditions on $V$ and $f$, Bieganowski and Secchi in [6] showed that the ground state solutions to (1.4) converge in $L_{\text {loc }}^{2}\left(\mathbb{R}^{N}\right)$. Using the penalized technique and the variational methods, An et al. in [3] proved the existence of a positive solution to (1.4) with the fast decaying potential $V$. In [10], Dávila, del Pino, and Wei generalized some previous results on the Schrödinger equation to fractional problem (1.4) by using the Lyapunov-Schmidt variational reduction. In [27], Long, Peng, and Yang obtained infinitely many non-radial positive solutions for (1.1) whose functional energy is very large. For the general nonlinearity, in [32], Secchi constructed solutions to (1.4), and the approach based on minimization on the Nehari manifold. For the critical case, He and Zou in [21] considered the following problem with critical growth:

$$
(-\Delta)^{s} u+u=u^{2_{s}^{*}-1}+\lambda(f(x, u)+h(x)), \quad u>0, x \in \mathbb{R}^{N},
$$

where the parameter $\lambda>0,2_{s}^{*}$ is the critical Sobolev exponent, $f$ and $h$ satisfy certain conditions. They showed the bifurcation and multiplicity of positive solutions for (1.5) in their paper. In [19], Guo and He proved the existence and concentration of positive solutions to the following fractional nonlinear Schrödinger equation:

$$
\epsilon^{2 s}(-\Delta)^{s} u+V(x) u=P(x) g(u)+Q(x) u^{2 *-2} u, \quad x \in \mathbb{R}^{N} .
$$

For the supercritical case, Ao et al. in [4] proved the existence of bound state solutions for (1.4). When the potential and nonlinearity satisfy certain conditions, Bisci and Rădulescu in [30] studied the existence of multiple ground state solutions for the following problem:

$$
(-\Delta)^{s} u+V(x) u=\lambda f(x, u)
$$

For other existence results, we refer to $[1,2,5,7-9,12,14,17,18,20,22,25,26,28,29,31$, 35-37] and the references therein.

After the above bibliography review, we want to specifically mention two papers [13] and [33]. In [33], Wei and Yan used a constructive method to produce infinitely many non-radial solutions to (1.1) when $s=1$ with high energy. Using the same method, Duan and Musso in [13] got other type of building blocks for the same problem in [33]. It is worth mentioning here that the structure of solutions in $[13,33]$ is dissimilar. Compared to the results in [33], the solutions in [13] have a more complex concentration structure. The solutions in [33] show the polygonal symmetry in the $\left(x_{1}, x_{2}\right)$-plane and are radially symmetric in other variables. However, the solutions in [13] have the polygonal symmetry in the $\left(x_{1}, x_{2}\right)$-plane, the even symmetry in the $x_{3}$ direction, and the radial symmetry in other variables. Inspired by the two works mentioned above, our aim is to construct new type solutions like the solutions in [13] which have a more complex concentration 
structure for problem (1.1). Since the fractional Laplacian operator is a nonlocal one, it is difficult to use the methods for local operator directly. For instance, the ground state for $-\Delta$ decays exponentially at infinity. In contrast, the ground state for (1.7) decays algebraically at infinity. So, the research of problem (1.1) becomes more complicated. At the same time, we need to redetermine the range for the parameters $h$ and $r$ which will be used to determine the position of the locations $\bar{y}_{j}, \underline{y}_{j}$ of the bumps in $W_{r, h}(x)$.

Next, we give some notation and definitions which are used in our paper.

Let $N \geq 3, m$ be an integer and introduce the points

$$
\left\{\begin{array}{l}
\bar{y}_{j}=r\left(\sqrt{1-h^{2}} \cos \frac{2(j-1) \pi}{k}, \sqrt{1-h^{2}} \sin \frac{2(j-1) \pi}{k}, h, \mathbf{0}\right), \quad j=1,2, \ldots, k, \\
\underline{y}_{j}=r\left(\sqrt{1-h^{2}} \cos \frac{2(j-1) \pi}{k}, \sqrt{1-h^{2}} \sin \frac{2(j-1) \pi}{k},-h, \mathbf{0}\right), \quad j=1,2, \ldots, k,
\end{array}\right.
$$

where $\mathbf{0}$ is the zero vector in $\mathbb{R}^{N-3}$.

Throughout this paper, we assume that $(r, h) \in \Lambda_{k}$ and define

$$
\begin{aligned}
\Lambda_{k}:= & {\left[\left(\left(\frac{\tilde{A}_{1}(N+2 s)}{E m}-\alpha\right)^{\frac{1}{N+2 s-m}}\right) k^{\frac{N+2 s}{N+2 s-m}},\left(\left(\frac{\tilde{A}_{1}(N+2 s)}{E m}+\alpha\right)^{\frac{1}{N+2 s+m}}\right) k^{\frac{N+2 s}{N+2 s-m}}\right] } \\
& \times\left[\left(\frac{A_{3}}{\tilde{A_{1}}}-\beta\right)^{\frac{1}{N+2 s+2}} \frac{1}{k^{\frac{N+2 s}{N+2 s+2}}},\left(\frac{A_{3}}{\tilde{A_{1}}}+\beta\right)^{\frac{1}{N+2 s+2}} \frac{1}{k^{N+2 s}}\right],
\end{aligned}
$$

where $\alpha, \beta>0$ are small constants, $\tilde{A}_{1}, A_{3}$, and $E$ are defined in Proposition 4.1.

Set $x=\left(x_{1}, x_{2}, x_{3}, x^{\prime \prime}\right) \in \mathbb{R} \times \mathbb{R} \times \mathbb{R} \times \mathbb{R}^{N-3}$. Define

$$
\begin{aligned}
\aleph_{s}= & \left\{u: u \in H^{s}\left(\mathbb{R}^{N}\right), u \text { is even in } x_{l}, l=2, \ldots, N,\right. \\
& u\left(\sqrt{x_{1}^{2}+x_{2}^{2}} \cos \theta, \sqrt{x_{1}^{2}+x_{2}^{2}} \sin \theta, x_{3}, x^{\prime \prime}\right) \\
& \left.=u\left(\sqrt{x_{1}^{2}+x_{2}^{2}} \cos \left(\theta+\frac{2 \pi j}{k}\right), \sqrt{x_{1}^{2}+x_{2}^{2}} \sin \left(\theta+\frac{2 \pi j}{k}\right), x_{3}, x^{\prime \prime}\right)\right\},
\end{aligned}
$$

where $\theta=\arctan \frac{x_{2}}{x_{1}}$.

We choose the unique positive solution $W$ (up to translations and dilations) of the following equation:

$$
\left\{\begin{array}{l}
(-\Delta)^{s} u+u=u^{p}, \quad u>0 \text { in } \mathbb{R}^{N} \\
u(0)=\max _{\mathbb{R}^{N}} u(x)
\end{array}\right.
$$

to construct the approximate solution for (1.1).

Denote

$$
W_{r, h}(x)=\sum_{j=1}^{k} W_{\bar{y}_{j}}+\sum_{j=1}^{k} W_{\underline{y}_{j}},
$$

where $W_{\bar{y}_{j}}=W\left(x-\bar{y}_{j}\right), W_{\underline{y}_{j}}=W\left(x-\underline{y}_{j}\right)$. 
For $j=1, \ldots, k$, we divide $\mathbb{R}^{N}$ into $k$ points:

$$
\begin{aligned}
\Omega_{j}:= & \left\{x=\left(x_{1}, x_{2}, x_{3}, x^{\prime \prime}\right) \in \mathbb{R}^{3} \times \mathbb{R}^{N-3}:\right. \\
& \left.\left\langle\frac{\left(x_{1}, x_{2}\right)}{\left|\left(x_{1}, x_{2}\right)\right|},\left(\cos \frac{2(j-1) \pi}{k}, \sin \frac{2(j-1) \pi}{k}\right)\right\} \geq \cos \frac{\pi}{k}\right\} .
\end{aligned}
$$

For $\Omega_{j}$, we divide it into two points:

$$
\begin{aligned}
& \Omega_{j}^{+}=\left\{x: x=\left(x_{1}, x_{2}, x_{3}, x^{\prime \prime}\right) \in \Omega_{j}, x_{3} \geq 0\right\}, \\
& \Omega_{j}^{-}=\left\{x: x=\left(x_{1}, x_{2}, x_{3}, x^{\prime \prime}\right) \in \Omega_{j}, x_{3}<0\right\} .
\end{aligned}
$$

We see that

$$
\mathbb{R}^{N}=\bigcup_{j=1}^{k} \Omega_{j}, \quad \Omega_{j}=\Omega_{j}^{+} \cup \Omega_{j}^{-}
$$

and the interior of

$$
\Omega_{j} \cap \Omega_{l}, \quad \Omega_{j}^{+} \cap \Omega_{j}^{-}
$$

and empty for $j \neq l$.

Before we give the main theorem, we first give the following conditions on the potential function $K$ :

$(K)$

$$
K(r)=1-\frac{a}{r^{m}}+O\left(\frac{1}{r^{m+\theta}}\right), \quad \text { as } r \rightarrow+\infty,
$$

for some $a>0, \theta>0$ and $\frac{N+2 s}{N+2 s+1}<m<N+2 s$.

Our main result is summarized as follows.

Theorem 1.1 Assume that $N \geq 3,0<s<1,1<p<\frac{N+2 s}{N-2 s}$. If $K(r)$ satisfies $K$, then there exists $k_{0}>0$ such that, for all integer $k>k_{0}$, problem (1.1) has a solution $U_{k}$ of the form

$$
U_{k}=W_{r, h}(x)+v_{r, h},
$$

where $v_{r, h} \in \aleph_{s},(r, h) \in \Lambda_{k}$, and as $k \rightarrow+\infty$,

$$
\int_{\mathbb{R}^{2 N}} \frac{\left|v_{r, h}(x)-v_{r, h}(y)\right|^{2}}{|x-y|^{N+2 s}}+\int_{\mathbb{R}^{N}} v_{r, h}^{2} \rightarrow 0
$$

In this paper, we first give some preliminaries in Sect. 2, and then we study the reduced finite dimensional problem in Sect. 3. In Sect. 4, we prove Theorem 1.1. Some useful lemmas are left in the Appendix. 


\section{Some preliminaries}

In this section, we list a couple of important properties and basic theory of fractional Sobolev spaces which are used in our paper. For more technical details, we refer the reader to [11].

For any $s \in(0,1)$, the space $H^{s}\left(\mathbb{R}^{N}\right)=W^{s, 2}\left(\mathbb{R}^{N}\right)$ is defined by

$$
\begin{aligned}
H^{s}\left(\mathbb{R}^{N}\right) & =\left\{u \in L^{2}\left(\mathbb{R}^{N}\right): \frac{|u(x)-u(y)|}{|x-y|^{\frac{N}{2}+s}} \in L^{2}\left(\mathbb{R}^{N} \times \mathbb{R}^{N}\right)\right\} \\
& =\left\{u \in L^{2}\left(\mathbb{R}^{N}\right): \int_{\mathbb{R}^{N}}\left(1+|\xi|^{2 s}\right)|\widehat{u}(\xi)|^{2}<\infty\right\} .
\end{aligned}
$$

The norm of $H^{s}\left(\mathbb{R}^{N}\right)$ is written as

$$
\|u\|_{s}=\sqrt{\langle u, u\rangle_{s}}, \quad u \in H^{s}\left(\mathbb{R}^{N}\right),
$$

where

$$
\begin{aligned}
\left\langle v_{1}, v_{2}\right\rangle_{s} & =\left\langle v_{1}, v_{2}\right\rangle+\int_{\mathbb{R}^{N}} v_{1} v_{2} \\
& :=\int_{\mathbb{R}^{N}} \int_{\mathbb{R}^{N}} \frac{\left(v_{1}(x)-v_{1}(y)\right)\left(v_{2}(x)-v_{2}(y)\right)}{|x-y|^{N+2 s}} d x d y+\int_{\mathbb{R}^{N}} v_{1} v_{2} .
\end{aligned}
$$

The following identity comes from Proposition 3.6 in [11]:

$$
[u]_{H^{s}\left(\mathbb{R}^{N}\right)}=C\left(\int_{\mathbb{R}^{N}}|\xi|^{2 s}|\widehat{u}(\xi)|^{2} d \xi\right)^{\frac{1}{2}}=C\left\|(-\Delta)^{\frac{s}{2}} u\right\|_{L^{2}\left(\mathbb{R}^{N}\right)},
$$

where is the Fourier transform, $C$ is a suitable positive constant depending only on $s$, and $[u]_{H^{s}\left(\mathbb{R}^{N}\right)}$ is the Gagliardo (semi) norm of the form

$$
[u]_{H^{s}\left(\mathbb{R}^{N}\right)}:=\langle u, u\rangle^{\frac{1}{2}}=\left(\int_{\mathbb{R}^{N}} \int_{\mathbb{R}^{N}} \frac{|u(x)-u(y)|^{2}}{|x-y|^{N+2 s}} d x d y\right)^{\frac{1}{2}} .
$$

The following results play a key role in proving Theorem 1.1.

Theorem 2.1 ([11]) The following embeddings are continuous:

(1) $H^{s}\left(\mathbb{R}^{N}\right) \hookrightarrow L^{q}\left(\mathbb{R}^{N}\right), 2 \leq q \leq \frac{2 N}{N-2 s}$, if $N>2 s$,

(2) $H^{s}\left(\mathbb{R}^{N}\right) \hookrightarrow L^{q}\left(\mathbb{R}^{N}\right), 2 \leq q \leq \infty$, if $N=2 s$.

Moreover, for any $R>0$ and $p \in\left[1,2_{s}^{*}\right)$, the embedding $H^{s}\left(B_{R}\right) \hookrightarrow L^{p}\left(B_{R}\right)$ is compact.

Theorem 2.2 ([15, 16]) Let $N \geq 1, s \in(0,1)$, and $1<p<\frac{N+2 s}{N-2 s}$. Then the ground state solution $W$ of (1.7) has the following properties:

(1) (Uniqueness) $W \in H^{s}\left(\mathbb{R}^{N}\right)$ is positive and unique up to translations and dilations.

(2) (Symmetry, regularity, and decay) W is radially symmetric and strictly decreasing in $|x|$. Moreover, the solution $W$ satisfies

$$
\frac{C_{1}}{1+|x|^{N+2 s}} \leq W \leq \frac{C_{2}}{1+|x|^{N+2 s}}, \quad x \in \mathbb{R}^{N}
$$

with some constants $C_{2} \geq C_{1}>0$. 
(3) (Non-degeneracy) The kernel of the linear operator $(-\Delta)^{s}+1-p|W|^{p-1}$ is spanned by $\left\{\partial_{x_{1}} W, \partial_{x_{2}} W, \ldots \partial_{x_{N}} W\right\}$.

\section{Variational reduction}

Let $\bar{Z}_{1 j}=\frac{\partial W_{\bar{x}_{j}}}{\partial r}, \underline{Z}_{1 j}=\frac{\partial W_{\underline{x}_{j}}}{\partial r}, \bar{Z}_{2 j}=\frac{\partial W_{\bar{x}_{j}}}{\partial h}, \underline{Z}_{2 j}=\frac{\partial W_{\underline{x}_{j}}}{\partial h}, j=1, \ldots, k$.

Define

$$
H_{s}=\left\{v \in \aleph_{s}: \sum_{j=1}^{k} \int_{\mathbb{R}^{N}} W_{\bar{x}_{j}}^{p-1} \bar{Z}_{l j} v=0 \text { and } \sum_{j=1}^{k} \int_{\mathbb{R}^{N}} W_{\underline{x}_{j}}^{p-1} \underline{Z}_{l j} v=0, l=1,2, j=1, \ldots, k\right\} .
$$

The energy functional corresponding to (1.1) is defined as

$$
I(u)=\frac{1}{2}\langle u, u\rangle+\frac{1}{2} \int_{\mathbb{R}^{N}} u^{2}-\frac{1}{p+1} \int_{\mathbb{R}^{N}} K(x)|u|^{p+1}
$$

Letting

$$
J(\phi)=I\left(W_{r, h}+\phi\right)=I\left(\sum_{j=1}^{k}\left(W_{\bar{y}_{j}}+W_{y_{j}}\right)+\phi\right), \quad \phi \in H_{s}
$$

we can expand $J(\phi)$ as follows:

$$
J(\phi)=J(0)+l(\phi)+\frac{1}{2} L(\phi)+R(\phi), \quad \forall \phi \in H_{s}
$$

where

$$
\begin{aligned}
& l(\phi)=\left\langle W_{r, h}, \phi\right\rangle+\int_{\mathbb{R}^{N}} W_{r, h} \phi-\int_{\mathbb{R}^{N}} K(x) W_{r, h}^{p} \phi, \\
& L(\phi)=\langle\phi, \phi\rangle+\int_{\mathbb{R}^{N}} \phi^{2}-\int_{\mathbb{R}^{N}} p K(x) W_{r, h}^{p-1} \phi^{2},
\end{aligned}
$$

and

$$
R(\phi)=-\frac{1}{p+1} \int_{\mathbb{R}^{N}} K(x)\left(\left(W_{r, h}+\phi\right)^{p+1}-W_{r, h}^{p+1}-(p+1) W_{r, h}^{p} \phi-\frac{1}{2}(p+1) W_{r, h}^{p-1} \phi^{2}\right) .
$$

The following result implies that $L$ is invertible in $H_{s}$.

Lemma 3.1 There exists an integer $k_{0}>0$ such that, for $k \geq k_{0}$, there is a constant $C>0$ independent of $k$, satisfying that, for any $(r, h) \in \Lambda_{k}$,

$$
\|L u\|_{s} \geq C\|u\|_{s}, \quad \forall u \in H_{s} .
$$

Proof We argue by contradiction. Suppose that there are $n \rightarrow+\infty, u_{n} \in H_{s},\left(r_{k}, h_{k}\right) \in \Lambda_{k}$ such that

$$
\left\|L u_{n}\right\|_{s}=o(1)\left\|u_{n}\right\|_{s}, \quad\left\|u_{n}\right\|_{s}^{2}=k
$$


By symmetry, we have

$$
\begin{aligned}
& k \int_{\Omega_{1}} \int_{\mathbb{R}^{N}} \frac{\left(u_{n}(x)-u_{n}(y)\right)(\psi(x)-\psi(y))}{|x+y|^{N+2 s}}+k \int_{\Omega_{1}} u_{n} \psi-k \int_{\Omega_{1}} p K(x) W_{r, h}^{p-1} u_{n} \psi \\
& \quad=o(1)\left\|u_{n}\right\|_{s}\|\psi\|_{s}=o(\sqrt{k})\|\psi\|_{s}, \quad \forall \psi \in H_{s} .
\end{aligned}
$$

In particular

$$
\int_{\Omega_{1}} \int_{\mathbb{R}^{N}} \frac{\left|u_{n}(x)-u_{n}(y)\right|^{2}}{|x-y|^{N+2 s}}+\int_{\Omega_{1}}\left|u_{n}\right|^{2}-k \int_{\Omega_{1}} p K(x) W_{r, h}^{p-1}\left|u_{n}\right|^{2}=o_{n}(1)
$$

and

$$
\int_{\Omega_{1}} \int_{\mathbb{R}^{N}} \frac{\left|u_{n}(x)-u_{n}(y)\right|^{2}}{|x-y|^{N+2 s}}+\int_{\Omega_{1}}\left|u_{n}\right|^{2}=1
$$

Let $\tilde{u}_{n}=\tilde{u}_{n}\left(x+\bar{y}_{1}\right)$, we can choose $R>0$ such that $B_{R}\left(\bar{y}_{1}\right) \subset \Omega_{1}$. Thus

$$
\int_{B_{R}(0)} \int_{\mathbb{R}^{N}} \frac{\left|\tilde{u}_{n}(x)-\tilde{u}_{n}(y)\right|^{2}}{|x-y|^{N+2 s}}+\int_{B_{R}(0)}\left|\tilde{u}_{n}\right|^{2} \leq 1 .
$$

So, we can conclude

$$
\tilde{u_{n}} \rightarrow \tilde{u}, \quad \text { weakly in } H^{s}\left(\mathbb{R}^{N}\right)
$$

and

$$
\tilde{u_{n}} \rightarrow \tilde{u}, \quad \text { strongly in } L_{\text {loc }}^{2}\left(\mathbb{R}^{N}\right)
$$

At the same time, we can obtain that $\tilde{u}$ is even in $x_{j}, j=2, \ldots, N$.

From the orthogonal conditions for functions of $H_{s}$

$$
\int_{\mathbb{R}^{N}} W_{\bar{x}_{1}}^{p-1} \bar{Z}_{11} u_{n}=0, \quad \int_{\mathbb{R}^{N}} W_{\underline{x}_{j}}^{p-1} \bar{Z}_{21} u_{n}=0,
$$

we can get

$$
\int_{\mathbb{R}^{N}} W^{p-1} \frac{\partial W}{\partial x_{1}} \tilde{u}_{n}=\int_{\mathbb{R}^{N}} W^{p-1} \frac{\partial W}{\partial x_{3}} \tilde{u}_{n}=0
$$

Letting $k \rightarrow+\infty$, we obtain

$$
\int_{\mathbb{R}^{N}} W^{p-1} \frac{\partial W}{\partial x_{1}} \tilde{u}=\int_{\mathbb{R}^{N}} W^{p-1} \frac{\partial W}{\partial x_{3}} \tilde{u}=0
$$

Now, we claim that $u$ satisfies

$$
(-\Delta)^{s} u+u-p W^{p-1} u=0, \quad \text { in } \mathbb{R}^{N} .
$$


Set

$$
\tilde{H}_{s}=\left\{\psi: \psi \in H^{s}\left(\mathbb{R}^{N}\right), \int_{\mathbb{R}^{N}} W^{p-1} \frac{\partial W}{\partial x_{1}} \psi=\int_{\mathbb{R}^{N}} W^{p-1} \frac{\partial W}{\partial x_{3}} \psi=0\right\} .
$$

For any $R>0$, let $\psi \in C_{0}^{\infty}\left(B_{R}(0)\right) \cap \widetilde{H}_{s}$ be any function satisfying that $\psi$ is even in $x_{j}$, $j=2, \ldots, N$. Then $\psi_{1}(x)=\psi\left(x-\bar{y}_{1}\right) \in B_{R}\left(\bar{y}_{1}\right)$. By Lemma 5.3, and inserting $\psi_{1}(x)$ into (3.2), we have

$$
\int_{\mathbb{R}^{N}} \int_{\mathbb{R}^{N}} \frac{\left(u_{n}(x)-u_{n}(y)\right)(\psi(x)-\psi(y))}{|x-y|^{N+2 s}}+\int_{\mathbb{R}^{N}} u \psi-\int_{\mathbb{R}^{N}} p W^{p-1} u \psi=0 .
$$

But (3.5) holds for $\psi=C_{1} \frac{\partial W}{\partial x_{1}}+C_{2} \frac{\partial W}{\partial x_{3}}$. Hence, (3.5) is true for any $\psi \in H^{s}\left(\mathbb{R}^{N}\right)$. Then, by orthogonal condition (3.4), $\psi=0$, and thus

$$
\int_{B_{R}\left(\bar{y}_{1}\right)} u_{n}^{2}=o(1)
$$

Thus, we can take $R>0$ large enough, then

$$
\begin{aligned}
o(1)= & \int_{\Omega_{1}} \int_{\mathbb{R}^{N}} \frac{\left|u_{n}(x)-u_{n}(y)\right|^{2}}{|x-y|^{N+2 s}}+\int_{\Omega_{1}} u_{n}^{2}-\int_{\Omega_{1}} p K(x) W_{r, h}^{p-1} u_{n}^{2} \\
= & \int_{\Omega_{1}} \int_{\mathbb{R}^{N}} \frac{\left|u_{n}(x)-u_{n}(y)\right|^{2}}{|x-y|^{N+2 s}}+\int_{\Omega_{1}} u_{n}^{2} \\
& -\int_{\Omega_{1} \backslash B_{R}\left(\bar{y}_{1}\right)} p K(x) W_{r, h}^{p-1} u_{n}^{2}-\int_{B_{R}\left(\bar{y}_{1}\right)} p K(x) W_{r, h}^{p-1} u_{n}^{2} \\
\geq & \frac{1}{2}\left(\int_{\Omega_{1}} \int_{\mathbb{R}^{N}} \frac{\left|u_{n}(x)-u_{n}(y)\right|^{2}}{|x-y|^{N+2 s}}+\int_{\Omega_{1}} u_{n}^{2}\right)+o_{R}(1)+o(1),
\end{aligned}
$$

which is a contradiction to (3.3).

Moreover, we give the estimate for $l$.

Lemma 3.2 For $(r, h) \in \Lambda_{k}$, if $k \geq k_{0}$ for some integer $k_{0}>0$, then

$$
\|l\|_{s} \leq C\left(k^{\frac{1}{2}}\left(\frac{k}{r \sqrt{1-h^{2}}}\right)^{\frac{N+2 s}{2}+\tau}+\frac{k^{\frac{1}{2}}}{r^{\frac{N+2 s}{2}+\tau}}\right)
$$

where $C>0$ is a constant independent of $k$ and $\tau>0$ small enough.

Proof Recall that

$$
\begin{aligned}
l(\phi) & =\left\langle W_{r, h}, \phi\right\rangle+\int_{\mathbb{R}^{N}} W_{r, h} \phi-\int_{\mathbb{R}^{N}} K(x) W_{r, h}^{p} \phi \\
& =\int_{\mathbb{R}^{N}} \sum_{j=1}^{k}\left(W_{\bar{y}_{j}}^{p}+W_{\underline{y}_{j}}^{p}\right) \phi-\int_{\mathbb{R}^{N}} K(x) W_{r, h}^{p} \phi \\
& =\int_{\mathbb{R}^{N}}\left(\sum_{j=1}^{k}\left(W_{\bar{y}_{j}}^{p}+W_{\underline{y}_{j}}^{p}\right)-W_{r, h}^{p}\right) \phi-\int_{\mathbb{R}^{N}}(K(x)-1) W_{r, h}^{p} \phi .
\end{aligned}
$$


Using the fact $\frac{N+2 s}{N+2 s+1}<m<N+2 s$, we can check that

$$
\begin{aligned}
\int_{\mathbb{R}^{N}}\left|(K(x)-1) W_{r, h}^{p} \phi\right| & \\
= & 2 k \int_{\mathbb{R}^{N}}\left|(K(x)-1) W_{\bar{y}_{1}}^{p} \phi\right| \\
\leq & C k\left(\int_{\mathbb{R}^{N}}|K(x)-1|^{\frac{p+1}{p}} W_{\bar{y}_{1}}^{p+1}\right)^{\frac{p}{p+1}}\left(\int_{\mathbb{R}^{N}}|\phi|^{p+1}\right)^{\frac{1}{p+1}} \\
\leq & C k\|\phi\|_{s}\left(\int_{B \frac{r}{2}\left(\bar{y}_{1}\right)}|K(x)-1|^{\frac{p+1}{p}} W_{\bar{y}_{1}}^{p+1}+\int_{\mathbb{R}^{N} \backslash B_{\frac{r}{2}}\left(\bar{y}_{1}\right)} W_{\bar{y}_{1}}^{p+1}\right)^{\frac{p}{p+1}} \\
\leq & C\|\phi\|_{s}\left(\frac{k}{r^{m}}+k\left(\int_{\frac{r}{2}}^{+\infty} \frac{1}{r^{(N+2 s)(p+1)}} r^{N-1} d r\right)^{\frac{p}{p+1}}\right) \\
\leq & C\left\|^{\prime} \phi\right\|_{s}\left(\frac{k}{r^{m}}+k \frac{1}{r^{(N+2 s) p-\frac{p}{p+1} N}}\right) \\
\leq & \frac{k^{\frac{1}{2}}}{r^{\frac{m}{2}+\tau}}\|\phi\|_{s}
\end{aligned}
$$

for some small constant $\tau>0$.

Since $m>\frac{N+2 s}{N+2 s+1}$, we can choose $\sigma$ satisfying

$$
(N+2 s)(p+1)-\frac{p+1}{p}\left(\frac{N+2 s}{2}+\sigma\right)>N, \quad \frac{p}{p+1}-\frac{1}{2}<\frac{m}{N+2 s-m} \sigma .
$$

Then it follows from Lemma 5.1 and Lemma 5.3 that

$$
\begin{aligned}
& {\left[\int_{\mathbb{R}^{N}}\left(\sum_{l \neq j} W_{\bar{y}_{l}}^{\frac{p}{2}} W_{\bar{y}_{j}}^{\frac{p}{2}}\right)^{\frac{p+1}{p}}\right]^{\frac{p}{p+1}}} \\
& \leq\left[C \sum_{l \neq j} \int_{\mathbb{R}^{N}} \frac{1}{\left(1+\left|y-\bar{y}_{l}\right|\right)^{\frac{(N+2 s)(p+1)}{2}}} \frac{1}{\left(1+\left|y-\bar{y}_{j}\right|\right)^{\frac{(N+2 s)(p+1)}{2}}}\right]^{\frac{p}{p+1}} \\
& \leq\left[C \sum _ { l \neq j } \frac { 1 } { | \overline { y } _ { l } - \overline { y } _ { j } | ^ { \frac { p + 1 } { p } ( \frac { N + 2 s } { 2 } + \sigma ) } } \int _ { \mathbb { R } ^ { N } } \left(\frac{1}{\left(1+\left|y-\bar{y}_{l}\right|\right)^{(N+2 s)(p+1)-\frac{p+1}{p}\left(\frac{N+2 s}{2}+\sigma\right)}}\right.\right. \\
& \left.+\frac{1}{\left.\left(1+\left|y-\bar{y}_{j}\right|\right)^{(N+2 s)(p+1)-\frac{p+1}{p}\left(\frac{N+2 s}{2}+\sigma\right)}\right)}\right]^{\frac{p}{p+1}} \\
& \leq C\left(\sum_{l \neq j} \frac{1}{\left|\bar{y}_{l}-\bar{y}_{j}\right|^{\frac{p+1}{p}\left(\frac{N+2 s}{2}+\sigma\right)}}\right)^{\frac{p}{p+1}} \\
& =C k^{\frac{p}{p+1}}\left(\sum_{j=2}^{k} \frac{1}{\left|\bar{y}_{1}-\bar{y}_{j}\right|^{\frac{N+2 s}{2}+\sigma}}\right) \\
& \leq C k^{\frac{p}{p+1}}\left(\frac{k}{r \sqrt{1-h^{2}}}\right)^{\frac{N+2 s}{2}+\sigma}
\end{aligned}
$$




$$
\leq C k^{\frac{1}{2}}\left(\frac{k}{r \sqrt{1-h^{2}}}\right)^{\frac{N+2 s}{2}+\tau}
$$

and

$$
\begin{aligned}
& {\left[\int_{\mathbb{R}^{N}}\left(\sum_{l \neq j} W_{\bar{y}_{l}}^{p-1} W_{\bar{y}_{j}}\right)^{\frac{p+1}{p}}\right]^{\frac{p}{p+1}} } \\
& \leq {\left[C \sum_{l \neq j} \int_{\mathbb{R}^{N}} \frac{1}{\left(1+\left|y-\bar{y}_{l}\right|\right)^{\frac{(N+2 s)(p-1)(p+1)}{p}}} \frac{1}{\left(1+\left|y-\bar{y}_{j}\right|\right)^{\frac{(N+2 s)(p+1)}{p}}}\right]^{\frac{p}{p+1}} } \\
& \leq {\left[C \sum_{l \neq j} \frac{1}{\left|\bar{y}_{l}-\bar{y}_{j}\right|^{\frac{p+1}{p}\left(\frac{N+2 s}{2}+\sigma\right)}} \int_{\mathbb{R}^{N}} \frac{1}{\left(1+\left|y-\bar{y}_{l}\right|\right)^{(N+2 s)(p+1)-\frac{p+1}{p}\left(\frac{N+2 s}{2}+\sigma\right)}}\right.} \\
&\left.+\frac{1}{\left(1+\left|y-\bar{y}_{j}\right|\right)^{(N+2 s)(p+1)-\frac{p+1}{p}\left(\frac{N+2 s}{2}+\sigma\right)}}\right]^{\frac{p}{p+1}} \\
& \leq C\left(\sum_{l \neq j} \frac{1}{\left|\bar{y}_{l}-\bar{y}_{j}\right|^{\frac{p+1}{p}\left(\frac{N+2 s}{2}+\sigma\right)}}\right)^{\frac{p}{p+1}} \\
&= C k^{\frac{p}{p+1}} \sum_{j=2}^{k} \frac{1}{\left|\bar{y}_{1}-\bar{y}_{j}\right|^{\frac{N+2 s}{2}+\sigma}} \\
& \leq C k^{\frac{1}{2}}\left(\frac{k}{r \sqrt{1-h^{2}}}\right)^{\frac{N+2 s}{2}+\tau}
\end{aligned}
$$

for some small constant $\tau>0$.

Similarly, we have

$$
\begin{aligned}
& {\left[\int_{\mathbb{R}^{N}}\left(\sum_{l \neq j} W_{\underline{y}_{l}}^{\frac{p}{2}} W_{\underline{y}_{j}}^{\frac{p}{2}}\right)^{\frac{p+1}{p}}\right]^{\frac{p}{p+1}} \leq C k^{\frac{1}{2}}\left(\frac{k}{r \sqrt{1-h^{2}}}\right)^{\frac{N+2 s}{2}+\tau},} \\
& {\left[\int_{\mathbb{R}^{N}}\left(\sum_{l \neq j} W_{\bar{y}_{l}}^{\frac{p}{2}} W_{\underline{y}_{j}}^{\frac{p}{2}}\right)^{\frac{p+1}{p}}\right]^{\frac{p}{p+1}} \leq C k^{\frac{1}{2}}\left(\frac{k}{r \sqrt{1-h^{2}}}\right)^{\frac{N+2 s}{2}+\tau},} \\
& {\left[\int_{\mathbb{R}^{N}}\left(\sum_{l \neq j} W_{\underline{y}_{l}}^{p-1} W_{\underline{y}_{j}}\right)^{\frac{p+1}{p}}\right]^{\frac{p}{p+1}} \leq C k^{\frac{1}{2}}\left(\frac{k}{r \sqrt{1-h^{2}}}\right)^{\frac{N+2 s}{2}+\tau},}
\end{aligned}
$$

and

$$
\left[\int_{\mathbb{R}^{N}}\left(\sum_{l \neq j} W_{\bar{y}_{l}}^{p-1} W_{\underline{y}_{j}}\right)^{\frac{p+1}{p}}\right]^{\frac{p}{p+1}} \leq C k^{\frac{1}{2}}\left(\frac{k}{r \sqrt{1-h^{2}}}\right)^{\frac{N+2 s}{2}+\tau} .
$$

\section{Combining the above estimates, we obtain}

$$
\int_{\mathbb{R}^{N}}\left(\sum_{j=1}^{k}\left(W_{\bar{y}_{j}}^{p}+W_{y_{j}}^{p}\right)-W_{r, h}^{p}\right) \phi
$$




$$
\begin{aligned}
& \leq\left\{\begin{array}{l}
C \sum_{l \neq j} \int_{\mathbb{R}^{N}} W_{\bar{y}_{l}}^{\frac{p}{2}} W_{\bar{y}_{j}}^{\frac{p}{2}} \phi+C \sum_{l \neq j} \int_{\mathbb{R}^{N}} W_{\underline{y}_{l}}^{\frac{p}{2}} W_{\underline{y}_{j}}^{\frac{p}{2}} \phi+C \sum_{l \neq j} \int_{\mathbb{R}^{N}} W_{\bar{y}_{l}}^{\frac{p}{2}} W_{\underline{y}_{j}}^{\frac{p}{2}} \phi, \\
\quad \text { if } 1<p \leq 2, \\
C \sum_{l \neq j} \int_{\mathbb{R}^{N}} W_{\bar{y}_{l}}^{p-1} W_{\bar{y}_{j}} \phi+C \sum_{l \neq j} \int_{\mathbb{R}^{N}} W_{\underline{y}_{l}}^{p-1} W_{\underline{y}_{j}} \phi+C \sum_{l \neq j} \int_{\mathbb{R}^{N}} W_{\bar{y}_{l}}^{p-1} W_{\underline{y}_{j}} \phi, \\
\quad \text { if } p>2,
\end{array}\right. \\
& \leq\left\{\begin{array}{l}
\left.C\left[\int_{\mathbb{R}^{N}}\left(\sum_{l \neq j} W_{\bar{y}_{l}}^{\frac{p}{2}} W_{\bar{y}_{j}}^{\frac{p}{2}}\right)^{\frac{p+1}{p}}\right)^{\frac{p}{p+1}}\right]\|\phi\|_{s}+C\left[\int_{\mathbb{R}^{N}}\left(\sum_{l \neq j} W_{\underline{y}_{l}}^{\frac{p}{2}} W_{\underline{y}_{j}}^{\frac{p}{2}}\right)^{\frac{p+1}{p}}\right]^{\frac{p}{p+1}}\|\phi\|_{s}+ \\
\quad+C\left[\int_{\mathbb{R}^{N}}\left(\sum_{l \neq j} W_{\bar{y}_{l}}^{\frac{p}{2}} W_{\underline{y}_{j}}^{\frac{p}{2}}\right)^{\frac{p+1}{p}}\right]^{\frac{p}{p+1}}\|\phi\|_{s}, \quad \text { if } 1<p \leq 2, \\
C\left[\int_{\mathbb{R}^{N}}\left(\sum_{l \neq j} W_{\bar{y}_{l}}^{p-1} W_{\bar{y}_{j}}\right)^{\frac{p+1}{p}}\right]^{\frac{p}{p+1}}\|\phi\|_{s}+C\left[\int_{\mathbb{R}^{N}}\left(\sum_{l \neq j} W_{\underline{y}_{l}}^{p-1} W_{\underline{y}_{-}}\right)^{\frac{p+1}{p}}\right]^{\frac{p}{p+1}}\|\phi\|_{s} \\
\quad+C\left[\int_{\mathbb{R}^{N}}\left(\sum_{l \neq j} W_{\bar{y}_{l}}^{p-1} W_{\underline{y}_{j}}\right)^{\frac{p+1}{p}}\right]\|\phi\|_{s}, \quad \text { if } p>2,
\end{array}\right. \\
& \leq C k^{\frac{1}{2}}\left(\frac{k}{r \sqrt{1-h^{2}}}\right)^{\frac{N+2 s}{2}+\tau}
\end{aligned}
$$

for some small constant $\tau>0$.

Inserting the above estimates into (3.6), we obtain the desired results.

The proof of the following significant Proposition 3.3 is the same as in [27], we only describe the content of it briefly.

Proposition 3.3 There exist $k_{0}>0$ and a constant $C>0$ independent of $k$, for any $k \geq k_{0}$, there is a $C^{1}$ map from $\Lambda_{k}$ to $\aleph_{s}: v=v(r, h)$ satisfying $v \in H_{s}$ and

$$
\left.J^{\prime}(v)\right|_{H_{s}}=0
$$

Moreover,

$$
\|v\|_{s} \leq C k^{\frac{1}{2}}\left(\left(\frac{k}{r}\right)^{\frac{N+2 s}{2}+\tau}+\frac{1}{r^{\frac{m}{2}+\tau}}\right)
$$

where $\tau>0$ is small enough.

\section{Proof of our main result}

In this part, we mainly give the estimate in Proposition 4.1 and show Theorem 1.1.

Proposition 4.1 It holds

$$
\begin{aligned}
I\left(W_{r, h}\right)= & \frac{p-1}{p+1} k \int_{\mathbb{R}^{3}} W^{p+1}+\frac{2 k}{p+1} \frac{B_{1}^{\prime}}{r^{m}}-k \frac{A_{3}}{(r h)^{N+2 s}}-k \tilde{A_{1}}\left(\frac{k}{r \sqrt{1-h^{2}}}\right)^{N+2 s} \\
& +k O\left(\frac{1}{(r h)^{N+2 s+\tau}}+\left(\frac{k}{r \sqrt{1-h^{2}}}\right)^{N+2 s+\tau}+\frac{1}{\left(r \sqrt{1-h^{2}}\right)^{m+\tau}}\right),
\end{aligned}
$$

where $B_{1}^{\prime}, A_{3}, \tilde{A_{1}}$ are some positive constants and $\tau>0$ is small enough.

Proof

$$
I\left(W_{r, h}\right)=\frac{1}{2}\left\langle W_{r, h}, W_{r, h}\right\rangle+\frac{1}{2} \int_{\mathbb{R}^{N}} W_{r, h}^{2}-\frac{1}{p+1} \int_{\mathbb{R}^{N}} K(x)\left|W_{r, h}\right|^{p+1} .
$$


By symmetry, we see

$$
\begin{aligned}
& \left\langle W_{r, h}, W_{r, h}\right\rangle+\int_{\mathbb{R}^{N}} W_{r, h}^{2} \\
& =\left\langle\sum_{j=1}^{k} W_{\bar{y}_{j}}, \sum_{j=1}^{k} W_{\bar{y}_{j}}\right\rangle+\int_{\mathbb{R}^{N}}\left|\sum_{j=1}^{k} W_{\bar{y}_{j}}\right|^{2} \\
& +\left\langle\sum_{j=1}^{k} W_{\underline{y}_{j}}, \sum_{j=1}^{k} W_{\underline{y}_{j}}\right\rangle+\int_{\mathbb{R}^{N}}\left|\sum_{j=1}^{k} W_{\underline{y}_{j}}\right|^{2} \\
& +2\left\langle\sum_{j=1}^{k} W_{\bar{y}_{j}}, \sum_{j=1}^{k} W_{y_{j}}\right\rangle+2 \int_{\mathbb{R}^{N}}\left(\sum_{j=1}^{k} W_{\bar{y}_{j}}\right)\left(\sum_{j=1}^{k} W_{\underline{y}_{j}}\right) \\
& =\sum_{l=1}^{k} \sum_{j=1}^{k} \int_{\mathbb{R}^{N}} W_{\bar{y}_{j}}^{p} W_{\bar{y}_{l}}+\sum_{l=1}^{k} \sum_{j=1}^{k} \int_{\mathbb{R}^{N}} W_{\underline{y}_{j}}^{p} W_{\underline{y}_{l}}+2 \sum_{l=1}^{k} \sum_{j=1}^{k} \int_{\mathbb{R}^{N}} W_{\bar{y}_{j}}^{p} w_{\underline{y}_{l}} \\
& =k \int_{\mathbb{R}^{N}} W^{p+1}+k \sum_{j=2}^{k} \int_{\mathbb{R}^{N}} W_{\bar{y}_{1}}^{p} W_{\bar{y}_{j}}+k \int_{\mathbb{R}^{N}} W^{p+1}+k \sum_{j=2}^{k} \int_{\mathbb{R}^{N}} W_{\underline{y}_{1}}^{p} W_{y_{j}} \\
& +2 k \sum_{j=1}^{k} \int_{\mathbb{R}^{N}} W_{\bar{y}_{1}}^{p} W_{\underline{y}_{j}} \\
& =2 k \int_{\mathbb{R}^{N}} W^{p+1}+2 k \sum_{j=2}^{k} \int_{\mathbb{R}^{N}} W_{\bar{y}_{1}}^{p} W_{\bar{y}_{j}}+2 k \sum_{j=1}^{k} \int_{\mathbb{R}^{N}} W_{\bar{y}_{1}}^{p} W_{\underline{y}_{j}} .
\end{aligned}
$$

Next, we estimate the term $\int_{\mathbb{R}^{N}} K(x)\left|W_{r, h}\right|^{p+1}$. Using the symmetry, we can compute

$$
\begin{aligned}
& \int_{\mathbb{R}^{N}} K(x)\left|W_{r, h}\right|^{p+1} \\
& =2 k \int_{\Omega_{1}^{+}} K(x)\left|W_{\bar{y}_{1}}+W_{\underline{y}_{1}}+\sum_{j=2}^{k} W_{\bar{y}_{j}}+\sum_{j=2}^{k} w_{\underline{y}_{j}}\right|^{p+1} \\
& =2 k \int_{\Omega_{1}^{+}} K(x) W_{\bar{y}_{1}}^{p+1}+2 k \int_{\Omega_{1}^{+}}(p+1) K(x) W_{\bar{y}_{1}}^{p}\left(W_{\underline{y}_{1}}+\sum_{j=2}^{k} W_{\bar{y}_{j}}+\sum_{j=2}^{k} W_{\underline{y}_{j}}\right) \\
& \quad+2 k\left\{\begin{array}{l}
O\left(\int_{\Omega_{1}^{+}} W_{\bar{y}_{1}}^{\frac{p+1}{2}}\left(W_{\bar{y}_{1}}+\sum_{j=2}^{k} W_{\bar{y}_{j}}+\sum_{j=2}^{k} w_{\underline{y}_{j}}\right)^{\frac{p+1}{2}}\right), \quad \text { if } 1<p \leq 2, \\
O\left(\int_{\Omega_{1}^{+}} W_{\bar{y}_{1}}^{p-1}\left(W_{\underline{y}_{1}}+\sum_{j=2}^{k} W_{\bar{y}_{j}}+\sum_{j=2}^{k} w_{\underline{y}_{j}}\right)^{2}\right), \quad \text { if } p>2 .
\end{array}\right.
\end{aligned}
$$

For $x \in \Omega_{1}^{+}$, we have $\left|x-\bar{y}_{j}\right| \geq \frac{1}{2}\left|\bar{y}_{j}-\bar{y}_{1}\right|$ and $\left|x-\bar{y}_{j}\right| \geq\left|x-\bar{y}_{1}\right|$. So

$$
\begin{aligned}
\sum_{j=2}^{k} W_{\bar{y}_{j}} & \leq C \sum_{j=2}^{k} \frac{1}{\left(1+\left|x-\bar{y}_{j}\right|\right)^{N+2 s-\gamma}} \frac{1}{\left(1+\left|x-\bar{y}_{j}\right|\right)^{\gamma}} \\
& \leq C \sum_{j=2}^{k} \frac{1}{\left(\left|\bar{y}_{1}-\bar{y}_{j}\right|\right)^{N+2 s-\gamma}} \frac{1}{\left(1+\left|x-\bar{y}_{1}\right|\right)^{\gamma}}
\end{aligned}
$$


and

$$
\begin{aligned}
\sum_{j=2}^{k} W_{y_{j}} & \leq C \sum_{j=2}^{k} \frac{1}{\left(1+\left|x-\underline{y}_{j}\right|\right)^{N+2 s-\gamma}} \frac{1}{\left(1+\left|x-\underline{y}_{j}\right|\right)^{\gamma}} \\
& \leq C \sum_{j=2}^{k} \frac{1}{\left(\left|\underline{y}_{1}-\underline{y}_{j}\right|\right)^{N+2 s-\gamma}} \frac{1}{\left(1+\left|x-\underline{y}_{1}\right|\right)^{\gamma}} .
\end{aligned}
$$

Here, we choose $\gamma>0$ satisfying

$$
\min \left\{\frac{p+1}{2}(N+2 s-\gamma), 2(N+2 s-\gamma)\right\}>N+2 s
$$

and the parameter $\gamma$ is useful in (4.4) and (4.5).

Using the fact that $W_{\bar{y}_{j}} \geq W_{y_{j}}$ for $x \in \Omega_{1}^{+},(4.2)$ and (4.3), we can check that

$$
\begin{aligned}
& \int_{\Omega_{1}^{+}} W_{\bar{y}_{1}}^{\frac{p+1}{2}}\left(W_{\underline{y}_{1}}+\sum_{j=2}^{k} W_{\bar{y}_{j}}+\sum_{j=2}^{k} W_{\underline{y}_{j}}\right)^{\frac{p+1}{2}} \\
& \leq C \int_{\Omega_{1}^{+}} W_{\bar{y}_{1}}^{\frac{p+1}{2}}\left(W_{\underline{y}_{1}}+\sum_{j=2}^{k} W_{\bar{y}_{j}}\right)^{\frac{p+1}{2}} \\
& \leq C \int_{\Omega_{1}^{+}} \frac{1}{\left(1+\left|x-\bar{y}_{1}\right|\right)^{\frac{(N+2 s)(p+1)}{2}}}\left(\frac{1}{\left(1+\left|x-\underline{y}_{1}\right|\right)^{N+2 s}}\right. \\
& \left.+\sum_{j=2}^{k} \frac{1}{\left(\left|\bar{y}_{1}-\bar{y}_{j}\right|\right)^{N+2 s-\gamma}} \frac{1}{\left(1+\left|x-\bar{y}_{1}\right|\right)^{\gamma}}\right)^{\frac{p+1}{2}} \\
& \leq C \int_{\Omega_{1}^{+}} \frac{1}{\left(1+\left|x-\bar{y}_{1}\right|\right)^{(N+2 s)\left(\frac{p+1}{2}-\tau\right)}} \frac{1}{\left(1+\left|x-\underline{y}_{1}\right|\right)^{\frac{(N+2 s)(p+1)}{2}}} \frac{1}{\left(1+\left|x-\bar{y}_{1}\right|\right)^{(N+2 s) \tau}} \\
& +C \int_{\Omega_{1}^{+}} \frac{1}{\left(1+\left|x-\bar{y}_{1}\right|\right)^{\frac{(N+2 s)(p+1)}{2}+\frac{p+1}{2} \gamma}}\left(\sum_{j=2}^{k} \frac{1}{\left(\left|\bar{y}_{1}-\bar{y}_{j}\right|\right)^{N+2 s-\gamma}}\right)^{\frac{p+1}{2}} \\
& \leq C \frac{1}{\left|\bar{y}_{1}-\underline{y}_{1}\right|^{N+2 s}} \int_{\Omega_{1}^{+}}\left(\frac{1}{\left(1+\left|x-\bar{y}_{1}\right|\right)^{(N+2 s)(p-\tau)}}+\frac{1}{\left(1+\left|x-\underline{y}_{1}\right|\right)^{(N+2 s)(p-\tau)}}\right) \\
& \times \frac{1}{\left(1+\left|x-\bar{y}_{1}\right|\right)^{(N+2 s) \tau}}+C\left(\sum_{j=2}^{k} \frac{1}{\left|\bar{y}_{1}-\bar{y}_{j}\right|^{N+2 s-\gamma}}\right)^{\frac{p+1}{2}} \\
& \leq \frac{C}{\left|\bar{y}_{1}-\underline{y}_{1}\right|^{N+2 s+\tau}}+C\left(\sum_{j=2}^{k} \frac{1}{\left|\bar{y}_{1}-\bar{y}_{j}\right|^{N+2 s-\gamma}}\right)^{\frac{p+1}{2}} \\
& \leq C\left(\frac{1}{r h}\right)^{N+2 s+\tau}+C\left(\frac{k}{r \sqrt{1-h^{2}}}\right)^{\frac{(N+2 s-\gamma)(p+1)}{2}} \\
& \leq C\left(\frac{1}{r h}\right)^{N+2 s+\tau}+C\left(\frac{k}{r \sqrt{1-h^{2}}}\right)^{N+2 s+\tau}
\end{aligned}
$$


and

$$
\begin{aligned}
& \int_{\Omega_{1}^{+}} W_{\bar{y}_{1}}^{p-1}\left(W_{\underline{y}_{1}}+\sum_{j=2}^{k} W_{\bar{y}_{j}}+\sum_{j=2}^{k} W_{\underline{y}_{j}}\right)^{2} \\
& \leq C \int_{\Omega_{1}^{+}} W_{\bar{y}_{1}}^{p-1}\left(W_{\underline{y}_{1}}+\sum_{j=2}^{k} W_{\bar{y}_{j}}\right)^{2} \\
& \leq C \int_{\Omega_{1}^{+}} \frac{1}{\left(1+\left|x-\bar{y}_{1}\right|\right)^{(N+2 s)(p-1-\tau)}} \frac{1}{\left(1+\left|x-\underline{y}_{1}\right|\right)^{2(N+2 s)}} \frac{1}{\left(1+\left|x-\bar{y}_{1}\right|\right)^{(N+2 s) \tau}} \\
& +C \int_{\Omega_{1}^{+}} \frac{1}{\left(1+\left|x-\bar{y}_{1}\right|\right)^{(N+2 s)(p-1)+2 \gamma}}\left(\sum_{j=2}^{k} \frac{1}{\left|\bar{y}_{1}-\bar{y}_{j}\right|^{N+2 s-\gamma}}\right)^{2} \\
& \leq C \frac{1}{\left|\bar{y}_{1}-\underline{y}_{1}\right|^{N+2 s}} \int_{\Omega_{1}^{+}}\left(\frac{1}{\left(1+\left|x-\bar{y}_{1}\right|\right)^{(N+2 s)(p-\tau)}}+\frac{1}{\left(1+\left|x-\underline{y}_{1}\right|\right)^{(N+2 s)(p-\tau)}}\right) \\
& \times \frac{1}{\left(1+\left|x-\bar{y}_{1}\right|\right)^{(N+2 s) \tau}}+C\left(\sum_{j=2}^{k} \frac{1}{\left|\bar{y}_{1}-\bar{y}_{j}\right|^{N+2 s-\gamma}}\right)^{2} \\
& \leq C\left(\frac{1}{r h}\right)^{N+2 s+\tau}+C\left(\frac{k}{r \sqrt{1-h^{2}}}\right)^{2(N+2 s-\gamma)} \\
& \leq C\left(\frac{1}{r h}\right)^{N+2 s+\tau}+C\left(\frac{k}{r \sqrt{1-h^{2}}}\right)^{N+2 s+\tau} \text {. }
\end{aligned}
$$

In addition,

$$
\begin{aligned}
& \int_{\Omega_{1}^{+}} K(x) W_{\bar{y}_{1}}^{p}\left(W_{\underline{y}_{1}}+\sum_{j=2}^{k} W_{\bar{y}_{j}}+\sum_{j=2}^{k} W_{\underline{y}_{j}}\right) \\
& =\int_{\Omega_{1}^{+}} W_{\bar{y}_{1}}^{p}\left(W_{\underline{y}_{1}}+\sum_{j=2}^{k} W_{\bar{y}_{j}}+\sum_{j=2}^{k} W_{\underline{y}_{j}}\right) \\
& \quad+\int_{\Omega_{1}^{+}}(K(x)-1) W_{\bar{y}_{1}}^{p}\left(W_{\underline{y}_{1}}+\sum_{j=2}^{k} W_{\bar{y}_{j}}+\sum_{j=2}^{k} W_{\underline{y}_{j}}\right) .
\end{aligned}
$$

Applying the estimates in Lemma 5.2, we get

$$
\begin{aligned}
\int_{\Omega_{1}^{+}} W_{\bar{y}_{1}}^{p}\left(W_{\underline{y}_{1}}+\sum_{j=2}^{k} W_{\bar{y}_{j}}+\sum_{j=2}^{k} W_{\underline{y}_{j}}\right) \\
=\int_{\mathbb{R}^{N}} W_{\bar{y}_{1}}^{p}\left(W_{\underline{y}_{1}}+\sum_{j=2}^{k} W_{\bar{y}_{j}}+\sum_{j=2}^{k} W_{\underline{y}_{j}}\right) \\
-\int_{\mathbb{R}^{N} \backslash \Omega_{1}^{+}} W_{\bar{y}_{1}}^{p}\left(W_{\underline{y}_{1}}+\sum_{j=2}^{k} W_{\bar{y}_{j}}+\sum_{j=2}^{k} W_{\underline{y}_{j}}\right) \\
=\frac{A_{3}}{(r h)^{N+2 s}}+\sum_{j=2}^{k} \frac{A_{1}}{\left|\bar{y}_{1}-\bar{y}_{j}\right|^{N+2 s}+\sum_{j=2}^{k} \frac{A_{2}}{\left|\bar{y}_{1}-\underline{y}_{j}\right|^{N+2 s}}}
\end{aligned}
$$


Lin and Diu Boundary Value Problems

(2021) 2021:58

Page 15 of 20

$$
\begin{aligned}
& +O\left(\frac{1}{(r h)^{N+2 s+\tau}}\right)+O\left(\left(\frac{k}{r \sqrt{1-h^{2}}}\right)^{N+2 s+\tau}\right) \\
& +O\left(\left(\frac{k}{r \sqrt{1-h^{2}}}\right)^{\sigma} \int_{\mathbb{R}^{N} \backslash \Omega_{1}^{+}} W_{\bar{y}_{1}}^{p-\sigma}\left(\sum_{j=2}^{k} W_{\bar{y}_{j}}+\sum_{j=2}^{k} W_{\underline{y}_{j}}\right)\right) \\
& +O\left(\frac{1}{(r h)^{\sigma}} \int_{\mathbb{R}^{N} \backslash \Omega_{1}^{+}} W_{\bar{y}_{1}}^{p-\sigma} W_{\underline{y}_{1}}\right) \\
& =\frac{A_{3}}{(r h)^{N+2 s}}+\sum_{j=2}^{k} \frac{A_{1}}{\left|\bar{y}_{1}-\bar{y}_{j}\right|^{N+2 s}}+\sum_{j=2}^{k} \frac{A_{2}}{\left|\bar{y}_{1}-\underline{y}_{j}\right|^{N+2 s}} \\
& +O\left(\frac{1}{(r h)^{N+2 s+\tau}}\right)+O\left(\left(\frac{k}{r \sqrt{1-h^{2}}}\right)^{N+2 s+\tau}\right),
\end{aligned}
$$

where $\sigma>0$ satisfies $p-\sigma>1$.

Moreover, we find

$$
\begin{aligned}
& \int_{\Omega_{1}^{+}}|K(x)-1| W_{\bar{y}_{1}}^{p}\left(W_{\underline{y}_{1}}+\sum_{j=2}^{k} W_{\bar{y}_{j}}+\sum_{j=2}^{k} W_{\underline{y}_{j}}\right) \\
& =\int_{\mathbb{R}^{N}}|K(x)-1| W_{\bar{y}_{1}}^{p}\left(W_{\underline{y}_{1}}+\sum_{j=2}^{k} W_{\bar{y}_{j}}+\sum_{j=2}^{k} W_{\underline{y}_{j}}\right) \\
& -\int_{\mathbb{R}^{N} \backslash \Omega_{1}^{+}}|K(x)-1| W_{\bar{y}_{1}}^{p}\left(W_{\underline{y}_{1}}+\sum_{j=2}^{k} W_{\bar{y}_{j}}+\sum_{j=2}^{k} W_{\underline{y}_{j}}\right) \\
& \leq \int_{B_{\frac{r \sqrt{1-h^{2}}}{2}}\left(\bar{y}_{1}\right)}|K(x)-1| W_{\bar{y}_{1}}^{p}\left(\sum_{j=2}^{k} W_{\bar{y}_{j}}+\sum_{j=2}^{k} W_{\underline{y}_{j}}\right) \\
& +\int_{B_{\frac{r}{2}\left(\bar{y}_{1}\right)}}|K(x)-1| W_{\bar{y}_{1}}^{p} W_{\underline{y}_{1}} \\
& +\int_{\mathbb{R}^{N} \backslash B_{\frac{r \sqrt{1-h^{2}}}{2}}\left(\bar{y}_{1}\right)}|K(x)-1| W_{\bar{y}_{1}}^{p}\left(\sum_{j=2}^{k} W_{\bar{y}_{j}}+\sum_{j=2}^{k} W_{\underline{y}_{j}}\right) \\
& +\int_{\mathbb{R}^{N} \backslash B \frac{r}{2}\left(\bar{y}_{1}\right)}|K(x)-1| W_{\bar{y}_{1}}^{p} W_{\underline{y}_{1}} \\
& +O\left(\left(\frac{k}{r \sqrt{1-h^{2}}}\right)^{N+2 s+\tau}\right)+O\left(\frac{1}{(r h)^{N+2 s+\tau}}\right) \\
& \leq \frac{C}{\left(r \sqrt{1-h^{2}}\right)^{m}} \int_{\mathbb{R}^{N}} W_{\bar{y}_{1}}^{p}\left(\sum_{j=2}^{k} W_{\bar{y}_{j}}+\sum_{j=2}^{k} W_{\underline{y}_{j}}\right)+\frac{C}{r^{m}} \int_{\mathbb{R}^{N}} W_{\bar{y}_{1}}^{p} W_{\underline{y}_{1}} \\
& +O\left(\frac{1}{\left(r \sqrt{1-h^{2}}\right)^{\sigma}} \sum_{j=2}^{k} \frac{1}{\left|\bar{y}_{1}-\bar{y}_{j}\right|^{N+2 s}} \int_{\mathbb{R}^{N} \backslash B_{\frac{r \sqrt{1-h^{2}}}{2}}\left(\bar{y}_{1}\right)}\left(W_{\bar{y}_{1}}^{p-\sigma}+W_{\bar{y}_{j}}^{p-\sigma}\right)\right) \\
& +O\left(\frac{1}{\left(r \sqrt{1-h^{2}}\right)^{\sigma}} \sum_{j=2}^{k} \frac{1}{\left|\bar{y}_{1}-\underline{y}_{j}\right|^{N+2 s}} \int_{\mathbb{R}^{N} \backslash B_{\frac{r \sqrt{1-h^{2}}}{2}}\left(\bar{y}_{1}\right)}\left(W_{\bar{y}_{1}}^{p-\sigma}+W_{\underline{y}_{j}}^{p-\sigma}\right)\right)
\end{aligned}
$$




$$
\begin{aligned}
& +O\left(\frac{1}{r^{\sigma}} \frac{1}{\left|\bar{y}_{1}-\underline{y}_{1}\right|^{N+2 s}} \int_{\mathbb{R}^{N} \backslash B_{\frac{r}{2}}\left(\bar{y}_{1}\right)}\left(W_{\bar{y}_{1}}^{p-\sigma}+W_{\underline{y}_{1}}^{p-\sigma}\right)\right) \\
& +O\left(\left(\frac{k}{r \sqrt{1-h^{2}}}\right)^{N+2 s+\tau}\right)+O\left(\frac{1}{(r h)^{N+2 s+\tau}}\right) \\
& =O\left(\left(\frac{k}{r \sqrt{1-h^{2}}}\right)^{N+2 s+\tau}\right)+O\left(\frac{1}{(r h)^{N+2 s+\tau}}\right) \\
& +O\left(\frac{1}{r^{m+\tau}}\right)+O\left(\frac{1}{\left(r \sqrt{1-h^{2}}\right)^{m+\tau}}\right) .
\end{aligned}
$$

Inserting (4.7), (4.8) into (4.6), we obtain

$$
\begin{aligned}
& \int_{\Omega_{1}^{+}} K(x) W_{\bar{y}_{1}}^{p}\left(W_{\underline{y}_{1}}+\sum_{j=2}^{k} W_{\bar{y}_{j}}+\sum_{j=2}^{k} W_{\underline{y}_{j}}\right) \\
& =\frac{A_{3}}{(r h)^{N+2 s}}+\sum_{j=2}^{k} \frac{A_{1}}{\left|\bar{y}_{1}-\bar{y}_{j}\right|^{N+2 s}}+\sum_{j=2}^{k} \frac{A_{2}}{\left|\bar{y}_{1}-\underline{y}_{j}\right|^{N+2 s}} \\
& +O\left(\frac{1}{(r h)^{N+2 s+\tau}}+\left(\frac{k}{r \sqrt{1-h^{2}}}\right)^{N+2 s+\tau}+\frac{1}{r^{m+\tau}}+\frac{1}{\left(r \sqrt{1-h^{2}}\right)^{m+\tau}}\right) .
\end{aligned}
$$

Similarly, we have

$$
\begin{aligned}
\int_{\Omega_{1}^{+}} K(x) W_{\bar{y}_{1}}^{p+1} \\
=\int_{\mathbb{R}^{N}} K(x) W_{\bar{y}_{1}}^{p+1}+O\left(\int_{\mathbb{R}^{N} \backslash B_{\frac{r \sqrt{1-h^{2}}}{k}}\left(\bar{y}_{1}\right)} K(x) W_{\bar{y}_{1}}^{p+1}\right) \\
=\int_{B_{\frac{r}{2}}\left(\bar{y}_{1}\right)} K(x) W_{\bar{y}_{1}}^{p+1}+\int_{\mathbb{R}^{N} \backslash B_{\frac{r}{2}}\left(\bar{y}_{1}\right)} K(x) W_{\bar{y}_{1}}^{p+1} \\
+O\left(\int_{\mathbb{R}^{N} \backslash B} \frac{r^{\frac{\sqrt{1-h^{2}}}{k}}}{\left.K(x) W_{1}\right)} W_{\bar{y}_{1}}^{p+1}\right) \\
=\int_{\mathbb{R}^{N}} W^{p+1}-\frac{B_{1}^{\prime}}{r^{m}}+O\left(\frac{1}{r^{m+\tau}}\right)+O\left(\int_{\frac{r \sqrt{1-h^{2}}}{k}}^{+\infty} \frac{1}{r^{(N+2 s)(p+1)}} r^{N-1} d r\right) \\
=\int_{\mathbb{R}^{N}} W^{p+1}-\frac{B_{1}^{\prime}}{r^{m}}+O\left(\frac{1}{r^{m+\tau}}\right)+O\left(\left(\frac{k}{r \sqrt{1-h^{2}}}\right)^{N+2 s-\tau}\right) .
\end{aligned}
$$

Combining (4.4)-(4.5) and (4.9)-(4.10), we deduce that

$$
\begin{aligned}
\int_{\mathbb{R}^{N}} K(x)\left|W_{r, h}\right|^{p+1} \\
=2 k\left(\int_{\mathbb{R}^{N}} W^{p+1}-\frac{B_{1}^{\prime}}{r^{m}}\right)+k O\left(\frac{1}{r^{m+\tau}}+\left(\frac{k}{r \sqrt{1-h^{2}}}\right)^{N+2 s-\tau}\right) \\
\quad+2 k(p+1)\left(\frac{A_{3}}{(r h)^{N+2 s}}+\sum_{j=2}^{k} \frac{A_{1}}{\left|\bar{y}_{1}-\bar{y}_{j}\right|^{N+2 s}}+\sum_{j=2}^{k} \frac{A_{2}}{\left|\bar{y}_{1}-\underline{y}_{j}\right|^{N+2 s}}\right)
\end{aligned}
$$




$$
\begin{aligned}
& +k O\left(\frac{1}{(r h)^{N+2 s+\tau}}+\left(\frac{k}{r \sqrt{1-h^{2}}}\right)^{N+2 s+\tau}\right) \\
& +k O\left(\frac{1}{r^{m+\tau}}+\frac{1}{\left(r \sqrt{1-h^{2}}\right)^{m+\tau}}\right) .
\end{aligned}
$$

Finally, by Lemma A.2 in [27], we have

$$
\begin{aligned}
I\left(W_{r, h}\right) & =\frac{1}{2}\left\langle W_{r, h}, W_{r, h}\right\rangle+\frac{1}{2} \int_{\mathbb{R}^{N}} W_{r, h}^{2}-\frac{1}{p+1} \int_{\mathbb{R}^{N}} K(x)\left|W_{r, h}\right|^{p+1} \\
= & k \int_{\mathbb{R}^{N}} W^{p+1}+k \sum_{j=2}^{k} \int_{\mathbb{R}^{N}} W_{\bar{y}_{1}}^{p} W_{\bar{y}_{j}}+k \sum_{j=2}^{k} \int_{\mathbb{R}^{N}} W_{\bar{y}_{1}}^{p} W_{\underline{y}_{j}} \\
& -\frac{2 k}{p+1}\left(\int_{\mathbb{R}^{N}} W^{p+1}-\frac{B_{1}^{\prime}}{r^{m}}\right)-2 k\left(\frac{A_{3}}{(r h)^{N+2 s}}+\sum_{j=2}^{k} \frac{A_{1}}{\left|\bar{y}_{1}-\bar{y}_{j}\right|^{N+2 s}}+\sum_{j=2}^{k} \frac{A_{2}}{\left|\bar{y}_{1}-\underline{y}_{j}\right|^{N+2 s}}\right) \\
+ & k O\left(\frac{1}{(r h)^{N+2 s+\tau}}+\left(\frac{k}{r \sqrt{1-h^{2}}}\right)^{N+2 s+\tau}+\frac{1}{r^{m+\tau}}+\frac{1}{\left(r \sqrt{1-h^{2}}\right)^{m+\tau}}\right) \\
= & \frac{p-1}{p+1} k \int_{\mathbb{R}^{N}} W^{p+1}+\frac{2 k}{p+1} \frac{B_{1}^{\prime}}{r^{m}} \\
& \left.-k\left(\frac{A_{3}}{(r h)^{N+2 s}}+\sum_{j=2}^{k} \frac{A_{1}}{\left|\bar{y}_{1}-\bar{y}_{j}\right|^{N+2 s}}+\sum_{j=2}^{k} \frac{A_{2}}{\left|\bar{y}_{1}-\underline{y}_{j}\right|^{N+2 s}}\right)^{N+2 s+\tau}+\frac{1}{\left(r \sqrt{1-h^{2}}\right)^{m+\tau}}\right) \\
& +k O\left(\frac{1}{(r h)^{N+2 s+\tau}}+\left(\frac{k}{r \sqrt{1-h^{2}}}\right)^{N+2 s}\right. \\
= & \frac{p-1}{p+1} k \int_{\mathbb{R}^{N}} W^{p+1}+\frac{2 k}{p+1} \frac{B_{1}^{\prime}}{r^{m}}-k \frac{A_{3}}{(r h)^{N+2 s}}-k \tilde{A}_{1}\left(\frac{k}{r \sqrt{1-h^{2}}}\right)^{N+2 s} \\
& +k O\left(\frac{1}{(r h)^{N+2 s+\tau}}+\left(\frac{k}{r \sqrt{1-h^{2}}}\right)^{N+2 s+\tau}+\frac{1}{\left(r \sqrt{1-h^{2}}\right)^{m+\tau}}\right) . \\
& k
\end{aligned}
$$

We are now to prove Theorem 1.1.

\section{Proof of Theorem 1.1}

$$
\begin{aligned}
F(r, h)= & I\left(W_{r, h}\right)+O\left(\|l\|_{s}\|v\|_{s}+\|v\|_{s}^{2}\right) \\
= & \frac{p-1}{p+1} k \int_{\mathbb{R}^{N}} W^{p+1}+\frac{2 k}{p+1} \frac{B_{1}^{\prime}}{r^{m}}-k \frac{A_{3}}{(r h)^{N+2 s}}-k \tilde{A_{1}}\left(\frac{k}{\sqrt{1-h^{2}}}\right)^{N+2 s} \\
& +k O\left(\frac{1}{(r h)^{N+2 s+\tau}}+\left(\frac{k}{r \sqrt{1-h^{2}}}\right)^{N+2 s}+\frac{1}{\left(r \sqrt{1-h^{2}}\right)^{m+\tau}}\right) \\
= & k\left(D+\frac{E}{r^{m}}-\frac{A_{3}}{(r h)^{N+2 s}}-\tilde{A_{1}}\left(\frac{k}{\sqrt{1-h^{2}}}\right)^{N+2 s}\right) \\
& +k O\left(\frac{1}{(r h)^{N+2 s+\tau}}+\left(\frac{k}{r \sqrt{1-h^{2}}}\right)^{N+2 s}+\frac{1}{\left(r \sqrt{1-h^{2}}\right)^{m+\tau}}\right),
\end{aligned}
$$

where $D=\frac{p-1}{p+1} \int_{\mathbb{R}^{N}} W^{p+1}, E=\frac{2 B_{1}^{\prime}}{p+1}$ and $A_{3}, \tilde{A_{1}}$ are constants defined in Proposition 4.1. 
Define

$$
G(r, h)=D+\frac{E}{r^{m}}-\frac{A_{3}}{(r h)^{N+2 s}}-\tilde{A_{1}}\left(\frac{k}{\sqrt{1-h^{2}}}\right)^{N+2 s}
$$

Then we consider the system

$$
\left\{\begin{array}{l}
G_{r}(r, h)=-E \frac{m}{r^{m}}+\frac{A_{3}(N+2 s)}{h^{N+2 s} r^{N+2 s+1}}+\frac{\tilde{A_{1}} k^{N+2 s}(N+2 s)}{\left(\sqrt{1-h^{2}}\right)^{N+2 s} r^{N+2 s+1}}=0 \\
G_{h}(r, h)=\frac{A_{3}(N+2 s)}{r^{N+2 s} h^{N+2 s+1}}-\frac{\tilde{A_{1}} k^{N+2 s}(N+2 s) h}{r^{N+2 s}\left(1-h^{2}\right)^{\frac{N+2 s+2}{2}}}=0 .
\end{array}\right.
$$

Then we can calculate that

$$
\begin{aligned}
& \tilde{r}=\left(\frac{\tilde{A}_{1}(N+2 s)}{E m}+o(1)\right)^{\frac{1}{N+2 s-m}} k^{\frac{N+2 s}{N+2 s+m}}, \\
& \tilde{h}=\left(\frac{A_{3}}{\tilde{A}_{1}}+o(1)\right)^{\frac{1}{N+2 s+2}} \frac{1}{k^{\frac{N+2 s}{N+2 s+2}}},
\end{aligned}
$$

which is really an interior point of $\Lambda_{k}$.

Define

$$
M(r, h)=\left[\begin{array}{ll}
G_{r r} & G_{r h} \\
G_{h r} & G_{h h}
\end{array}\right]
$$

By computing, we know

$$
\left.G_{r r}\right|_{(r, h)=(\tilde{r}, \tilde{h})}<0,\left.\quad G_{h h}\right|_{(r, h)=(\tilde{r}, \tilde{h})}<0
$$

and

$$
G_{r r} \times\left. G_{h h}\right|_{(r, h)=(\tilde{r}, \tilde{h})}-\left.G_{r h}^{2}\right|_{(r, h)=(\tilde{r}, \tilde{h})}>0 .
$$

So, we obtain that $(\tilde{r}, \tilde{h})$ is a maximum point of $G(r, h)$. Then the maximum of $G(r, h)$ in $\Lambda_{k}$ can be achieved. Thus, we can find the critical point $\left(r_{k}, h_{k}\right) \in \Lambda_{k}$ of $F(r, h)$. Through the conventional conclusion in [10], we can show that $W_{r_{k}, h_{k}}(x)+v_{r_{k}, h_{k}}$ is a critical point of $J$, then $W_{r_{k}, h_{k}}(x)+v_{r_{k}, h_{k}}$ is a solution of (1.1).

\section{Appendix}

Some vital lemmas are given as follows.

Lemma 5.1 (Lemma B.1, [34]) For any constant $0<\sigma \leq \min \{\alpha, \beta\}$, there is a constant $C>0$ such that

$$
\frac{1}{\left(1+\left|x-y_{j}\right|\right)^{\alpha}} \frac{1}{\left(1+\left|x-y_{l}\right|\right)^{\beta}} \leq \frac{C}{\left|y_{j}-y_{l}\right|^{\sigma}}\left(\frac{1}{\left(1+\left|x-y_{j}\right|\right)^{\alpha+\beta-\sigma}}+\frac{1}{\left(1+\left|x-y_{l}\right|\right)^{\alpha+\beta-\sigma}}\right)
$$

where $\alpha, \beta \geq 1$ are two constants. 
The proofs of the following two lemmas are similar to Lemma A.2 and (28) in [20] respectively.

Lemma 5.2 For $j=2,3, \ldots, k$, there exists a small constant $\tau>0$ such that

$$
\begin{aligned}
& \int_{\mathbb{R}^{N}} W_{\bar{y}_{1}}^{p} W_{\bar{y}_{j}}=\frac{A_{1}}{\left|\bar{y}_{1}-\bar{y}_{j}\right|^{N+2 s}}+O\left(\frac{1}{\left|\bar{y}_{1}-\bar{y}_{j}\right|^{N+2 s+\tau}}\right), \\
& \int_{\mathbb{R}^{N}} W_{\bar{y}_{1}}^{p} W_{y_{j}}=\frac{A_{2}}{\left|\bar{y}_{1}-y_{j}\right|^{N+2 s}}+O\left(\frac{1}{\left|\bar{y}_{1}-\underline{y}_{j}\right|^{N+2 s+\tau}}\right),
\end{aligned}
$$

and

$$
\int_{\mathbb{R}^{N}} W_{\bar{y}_{1}}^{p} W_{\underline{y}_{1}}=\frac{A_{3}}{(r h)^{N+2 s}}+O\left(\frac{1}{(r h)^{N+2 s+\tau}}\right)
$$

where $\tau>0$ is small enough.

Lemma 5.3 For $(r, h) \in \Lambda_{k}$ and $\eta \in(1, N+2 s]$, there is a constant $C>0$ such that

$$
\sum_{j=2}^{k} W_{\bar{y}_{j}} \leq C \frac{1}{\left(1+\left|x-\bar{y}_{1}\right|\right)^{N+2 s-\eta}} \frac{k^{\eta}}{\left|\bar{y}_{1}\right|^{\eta}} \leq C \frac{k^{\eta}}{\left|\bar{y}_{1}\right|^{\eta}} \text { for all } x \in \Omega_{1}^{+}
$$

and

$$
\sum_{j=2}^{k} W_{\underline{y}_{j}} \leq C \frac{1}{\left(1+\left|x-\underline{y}_{1}\right|\right)^{N+2 s-\eta}} \frac{k^{\eta}}{\left|\underline{y}_{1}\right|^{\eta}} \leq C \frac{k^{\eta}}{\left|\underline{y}_{1}\right|^{\eta}} \quad \text { for all } x \in \Omega_{1}^{+} .
$$

\section{Acknowledgements}

The authors wish to express their gratitude to the editors and the reviewers for the helpful comments.

\section{Funding}

The authors are partially supported by the Mathematics Tianyuan Foundation of National Natural Science Foundation of China (12026246), the National Natural Science Foundation of China (11601139), the Natural Science Foundation of Hubei Province (2019CFB522), and by the Scientific Research and Innovation Team of Hubei Normal University (2019CZ010).

Availability of data and materials

No data were used to support this study.

\section{Competing interests}

The authors declare that they have no competing interests.

\section{Authors' contributions}

The idea of this research was introduced by Weiming Liu. All authors contributed to the main results. All authors read and approved the final manuscript.

\section{Publisher's Note}

Springer Nature remains neutral with regard to jurisdictional claims in published maps and institutional affiliations.

Received: 30 March 2021 Accepted: 10 June 2021 Published online: 16 June 2021

\section{References}

1. Ambrosio, V:: Multiplicity of solutions for fractional Schrödinger systems in $\mathbb{R}^{N}$. Complex Var. Elliptic Equ. 65, 856-885 (2020)

2. Ambrosio, V., Figueiredo, G.: Ground state solutions for a fractional Schrödinger equation with critical growth Asymptot. Anal. 105, 159-191 (2017)

3. An, X., Duan, L., Peng, Y.: Semi-classical analysis for fractional Schrödinger equations with fast decaying potentials. Appl. Anal. https://doi.org/10.1080/00036811.2021.1880571 
4. Ao, W., Chan, H., González, M., Wei, J.: Bound state solutions for the supercritical fractional Schrödinger equation. Nonlinear Anal. 193, 111448 (2020)

5. Appolloni, L., Secchi, S.: Normalized solutions for the fractional NLS with mass supercritical nonlinearity. arXiv:2006.00239v2

6. Bieganowski, B., Secchi, S.: Non-local to local transition for ground states of fractional Schrödinger equations on $\mathbb{R}^{N}$. J. Fixed Point Theory Appl. 22, 76 (2020)

7. Cardoso, J.A., dos Prazeres, D.S., Severo, U.B.: Fractional Schrödinger equations involving potential vanishing at infinity and super-critical exponents. Z. Angew. Math. Phys. 71, 129 (2020)

8. Chen, J., Gao, Z:: Ground state solutions for fractional Schrödinger equation with variable potential and Berestycki-Lions type nonlinearity. Bound. Value Probl. 2019, 148 (2019). https://doi.org/10.1186/s13661-019-1260-7

9. Cheng, Z., Gui, C., Hu, Y.: Existence of solutions to the supercritical Hard-Littlewood-Sobolev system with fractional Laplacians. Discrete Contin. Dyn. Syst. 39, 1345-1358 (2019)

10. Dávila, J., Del Pino, M., Wei, J.: Concentrating standing waves for the fractional nonlinear Schrödinger equation. J. Differ. Equ. 256, 858-892 (2014)

11. Di Nezza, E., Palatucci, G., Valdinoci, E.: Hitchhiker's guide to the fractional Sobolev spaces. Bull. Sci. Math. 136 521-573 (2012)

12. Dinh, V.: On instability of standing waves for the mass-supercritical fractional nonlinear Schrödinger equation. Z. Angew. Math. Phys. 70, 58 (2019)

13. Duan, L., Musso, M.: New type of solutions for the nonlinear Schrödinger equation in $\mathbb{R}^{N}$. arXiv:2006.16125v1

14. Felmer, P., Quaas, A., Tan, J.: Positive solutions of the nonlinear Schrödinger equation with the fractional Laplacian. Proc. R. Soc. Edinb., Sect. A 142, 1237-1262 (2012)

15. Frank, R., Lenzmann, E.: Uniqueness and nondegeneracy of ground states for $(-\Delta)^{s} Q+Q-Q^{\alpha+1}=0$ in $\mathbb{R}$. Acta Math. 210, 261-318 (2013)

16. Frank, R., Lenzmann, E., Silvestre, L.: Uniqueness of radial solutions for the fractional Laplacian. Commun. Pure Appl. Math. 69, 1671-1726 (2016)

17. Gu, G., Tang, X., Zhang, Y.: Existence of positive solutions for a class of critical fractional Schrödinger-Poisson system with potential vanishing at infinity. Appl. Math. Lett. 99, 105984 (2020)

18. Guan, W., Wang, D., Hao, X.: Infinitely many solutions for a class of sublinear fractional Schrödinger equations with indefinite potentials. J. Inequal. Appl. 2020,61 (2020)

19. Guo, Q., He, X:: Semiclassical states for fractional Schrödinger equations with critical growth. Nonlinear Anal. 151, 164-186 (2017)

20. Guo, Y., Liu, T., Nie, J.: Solutions for fractional Schrödinger equation involving critical exponent via local Pohozaev identities. Adv. Nonlinear Stud. 20, 185-211 (2020)

21. He, X., Zou, W.: Bifurcation and multiplicity of positive solutions for nonhomogeneous fractional Schrödinger equations with critical growth. Sci. China Math. 63, 1571-1612 (2020)

22. Huang, W., Wang, L.: Ground state solutions of Nehari-Pohozaev type for a fractional Schrödinger-Poisson system with critical growth. Acta Math. Sci. 40B(4), 1064-1080 (2020)

23. Laskin, N.: Fractional quantum mechanics and Lévy path integrals. Phys. Lett. A 268, 29-305 (2000)

24. Laskin, N.: Fractional Schrödinger equation. Phys. Rev. E 66, 31 (2002)

25. Li, M., He, J., Xu, H., Yang, M.: Normalized solutions for a coupled fractional Schrödinger system in low dimensions. Bound. Value Probl. 2020, 166 (2020). https://doi.org/10.1186/s13661-020-01463-9

26. Li, Q. Teng, K., Wang, W. Zhang, J. Existence of nontrivial solutions for fractional Schrödinger equations with electromagnetic fields and critical or supercritical nonlinearity. Bound. Value Probl. 2020, 112 (2020). https://doi.org/10.1186/s13661-020-01409-1

27. Long, W., Peng, S., Yang, J.: Infinitely many positive and sign-changing solutions for nonlinear fractional scalar field equations. Discrete Contin. Dyn. Syst. 36, 917-939 (2016)

28. Luo, H., Zhang, Z.: Normalized solutions to the fractional Schrödinger equations with combined nonlinearities. Calc. Var. 59, 1-13 (2020)

29. Mingione, G., Rădulescu, V:: Recent developments in problems with nonstandard growth and nonuniform ellipticity. J. Math. Anal. Appl. 501, 125197 (2021). https://doi.org/10.1016/j.jmaa.2021.125197

30. Molica Bisci, G., Rădulescu, V.: Ground state solutions of scalar field fractional Schrödinger equations. Calc. Var. Partial Differ. Equ. 54, 2985-3008 (2015)

31. Palatucci, G., Pisante, A.: Improved Sobolev embeddings, profile decomposition and concentration-compactness for fractional Sobolev spaces. Calc. Var. Partial Differ. Equ. 50, 799-829 (2014)

32. Secchi, S.: Ground state solutions for nonlinear fractional Schrödinger equations in $\mathbb{R}^{N}$. J. Math. Phys. 54, 031501 (2013)

33. Wei, J., Yan, S.: Infinite many positive solutions for the nonlinear Schrödinger equation in $\mathbb{R}^{N}$. Calc. Var. Partial Differ. Equ. 37, 423-439 (2010)

34. Wei, J., Yan, S.: Infinite many positive solutions for the prescribed scalar curvature problem on $\mathbb{S}^{N}$. J. Funct. Anal. 258 3048-3081 (2010)

35. Xiang, M., Rădulescu, V.D., Zhang, B.: Nonlocal Kirchhoff problems with singular exponential nonlinearity. Appl. Math. Optim. (2021), in press. https://doi.org/10.1007/s00245-020-09666-3

36. Zhang, H., Zhang, F.: Multiplicity of semiclassical states for fractional Schrödinger equations with critical frequency. Nonlinear Anal. 190, 111599 (2020)

37. Zhang, P., Han, Z.: Existence of solutions for a nonhomogeneous sublinear fractional Schrödinger equation. Complex Var. Elliptic Equ. https://doi.org/10.1080/17476933.2021.1882438 